\title{
SUL LUOGO DEI PUNTI DI CONTATTO DELLE IPERSUPERFICIE DI DUE DATI SISTEMI LINEARI.
}

\author{
Nota di Giovanni Z. Giambelli (Torino).
}

Adunanza del 24 marzo I9o7.

In questa breve Nota mi propongo di mostrare, come una conveniente interpretazione analitica permetta di enunciare subito alcuni teoremi sulla varietà luogo dei punti di contatto di due ipersuperficie di due dati sistemi lineari. Particolarizzando i nostri risultati, si ottengono teoremi noti, dedotti però in modo diverso e meno rapido del nostro; per brevità si mostrerà solo, come dai nostri teoremi si deducano quelli del lavoro di P. Lorenzola *), perchè questo lavoro si può considerare come il più importante di quelli $\left.{ }^{* *}\right)$, in cui si studiano casi particolari della nostra questione.

Si designerd con $V_{i}\left(p_{0}, p_{1}, \ldots, p_{r}\right)$ la funzione simmetrica caratteristica $\left.{ }^{* * *}\right)$, che si deduce dallo sviluppo $\left(p_{0}+p_{1}+\cdots+p_{r}\right)^{i}$, quando in luogo di ciascuno dei coefficienti polinomiali si ponga l'unità, ossia la funzione aleph di WronsKI di ordine $i$ nelle $p_{0}, p_{1}, \ldots, p_{r}$. Col simbolo $\Delta_{(y, x)}$ si designerà l'espressione simbolica (operazione di polare)

e si porrà

$$
\sum_{i=0}^{i=d} y_{i} \frac{\partial}{\partial x_{i}}
$$

$$
\nabla_{r}^{(i, x)}=\frac{\mathrm{I}}{r !}\left[\mathbf{\Delta}_{(x, y)}\right]^{r}
$$

rispetto a questi simboli occorre aver presente quanto si è detto nel $\$ I del mio lavoro: Le varietà rappresentate per mezzo di una matrice generica di forme e le varietà generate

*) Sul luogo dei punti di contatto degli iperpiani passanti per un dato spazio lineare e tangenti alle forme di un dato sistema lineare [Giornale di Matematiche, tomo XLIII (1905), pp. 213-240].

**) Gli altri lavori si riferiscono allo spazio ordinario, tra i quali la Nota di C. Minko [questi Rendiconti, tomo XVII (1903), pp. 297-310] e quelle di L. Lo Monaco AprILe [questi Rendiconti, tomo XVIII (I904), pp. I-1; e pp. 164-I84]; alcuni risultati di queste tre Note si possono ottenere subito per mezzo di una conveniente interpretazione analitica, come segue dalla Nota di M. STuYvarRT, Sur la courbe lieu des points de contact des surfaces de deux faisceaux [questi Rendiconti, tomo XVIII (1904), pp. 294-300].

***) Cfr. per es. il $\$ I della mia Nota: Alcune proprietà delle funzioni simmetriche caratteristiche [Atti della R. Accademia delle Scienze di Torino, tomo XXXVIII (1903), pp. 823-844]. 
da sistemi lineari proiettivi di forme [Rend. R. Acc. Lincei, serie V, tomo XIV (I905)]. Inoltre colla locuzione "matrice nulla" s'intenderà che siano nulli tutti $\mathrm{i}$ determinanti di ordine massimo contenuti nella matrice.

Scrivendo il sistema di equazioni rappresentante la condizione, affinchè si tocchino in un punto due ipersuperficie di due dati sistemi lineari, e ricordando l'importante formola di S. RoBERTS sull'ordine della varietà rappresentata coll'annullare una data matrice di forme ${ }^{*}$ ), segue:

Teorema I. - Siano

$$
\left.\begin{array}{l}
\lambda_{0} F_{0}+\lambda_{1} F_{1}+\cdots+\lambda_{b} F_{b}=0 \\
\mu_{0} \varphi_{0}+\mu_{1} \varphi_{1}+\cdots+\mu_{k} \varphi_{k}=0
\end{array} \quad \text { (essendo o }<h, o<k, b+k<n\right)
$$

due sistemi lineari di ipersuperficie dello spazio fondamentale $[n]$. Le $F_{i}(i=0, \mathrm{I}, \ldots, b)$ siano forme di grado $p$, e le $\varphi_{i}(i=0, \mathrm{I}, \ldots, k)$ di grado $q$ nelle $x_{0}, x_{1}, \ldots, x_{n}$, coordinate correnti omogenee di punto nello spazio fondamentale $[n]$. La varietà $W$, luogo dei punti di contatto delle ipersuperficie del sistema lineare (I) colle ipersuperficie del sistema lineare (2), è rappresentata analiticamente annullando la matrice

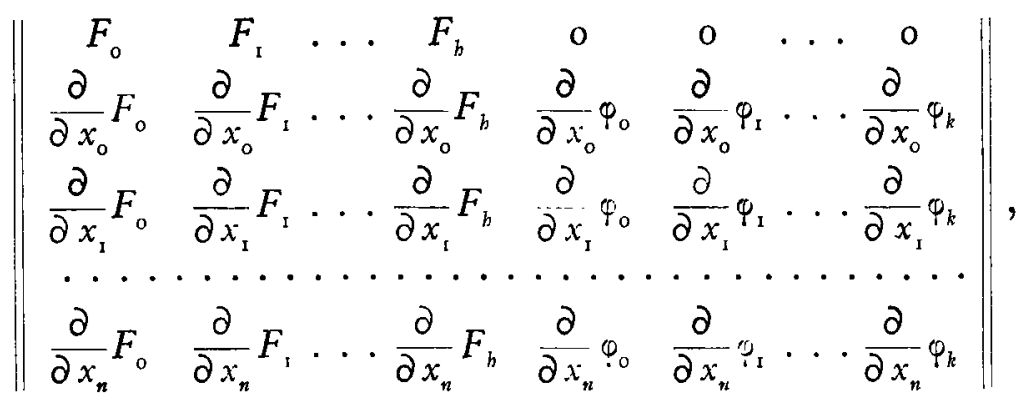

ed $\mathrm{e}$ di ordine

$$
\begin{aligned}
& \quad \sum_{i=0}^{i=n-k=k+1}\left(\begin{array}{c}
b+i \\
b
\end{array}\right)\left(\begin{array}{c}
n-b-i+\mathrm{I} \\
k
\end{array}\right)(p-\mathrm{I})^{i}(q-\mathrm{I})^{n-h-k-i+1} \\
& +\sum_{i=0}^{i=n-h-k}\left(\begin{array}{c}
b+i \\
b
\end{array}\right)\left(\begin{array}{c}
n-b-i \\
k
\end{array}\right)(p-\mathrm{I})^{i}(q-\mathrm{I})^{n-h-k-i}
\end{aligned}
$$

E necessario supporre $o<b, o<k$, perchè, se per es. fosse $k=0$, essendo $b>0$, allora coll'annullare la matrice (3) si rappresenta la várietà, che si spezza nella $W$ e nella varietà $W^{\prime}$, luogo dei punti doppi delle ipersuperficie del sistema lineare (I), ossia varietà Jacobiana del sistema lineare (I). Per ottenere in questo caso l'ordine della $W$ si dovrà togliere dalla formola data dal teorema I il termine

$$
\left(\begin{array}{c}
n+\mathrm{I} \\
b
\end{array}\right)(p-\mathrm{I})^{n-h+1}
$$

*) Sur l'ordre des conditions de la coexistence des équations algébriques a plusieurs variables [Journ. für Math. t. LXVII (1867), pp. 266-278]. Questa formola di RobERTs è un caso particołare delle formole contenute nella mia Memoria: Ordine di una varietà più ampia di quella rappresentata coll'annullare tutti $i$ minori di dato ordine estratti da una data matrice generica di forme [Mem. R. Istituto Lombardo, serie III, tomo XI (I904), pp. IOI-I33]. 
che è l'ordine della $W^{\prime}$, essendo la $W^{\prime}$ rappresentata coll'annullare la matrice

$$
\left\|\frac{\partial}{\partial x_{i}} F_{j}\right\| \quad(i=0, \mathrm{I}, \ldots, n ; j=0, \mathrm{I}, \ldots, b) .
$$

Teorema II. - La $W$ sega la varietà base del sistema lineare (I) nella varietà di ordine

$$
p^{h+1} \sum_{i=0}^{i=n-h-k}\left(\begin{array}{c}
b+i \\
b
\end{array}\right)\left(\begin{array}{c}
n-b-i \\
k
\end{array}\right)(p-1)^{i}(q-\mathrm{I})^{n-h-k-i},
$$

rappresentata analiticamente coll'annullare

$$
\begin{array}{lll}
\text { le } F_{0}, & F_{1}, \ldots, F_{b}, & \text { se } b+k=n, \\
\text { le } F_{0}, & F_{1} . \ldots, F_{h} \text { e la matrice }(4), & \text { se } b+k<n,
\end{array}
$$

dove con (4) s'indica la matrice, che si ottiene dalla (3) togliendo la prima linea.

La $W$ sega la varietà base del sistema lineare (2) nella varietà di ordine

$$
q^{k+1} \sum_{i=0}^{i=n-b-k}\left(\begin{array}{c}
b+i \\
b
\end{array}\right)\left(\begin{array}{c}
n-b-i \\
k
\end{array}\right)(p-1)^{i}(q-1)^{n-b-k-i},
$$

rappresentata analiticamente coll'annullare

$$
\begin{array}{ll}
\text { le } \varphi_{0}, \varphi_{1}, \ldots, \varphi_{k}, & \text { se } b+k=n \\
\text { le } \varphi_{0}, \varphi_{1}, \ldots, \varphi_{k} \text { e la matrice (4), } & \text { se } b+k<n .
\end{array}
$$

Ricordando le proprieta elementari del simbolo $\nabla_{r}^{(y, x)}$ segue:

Teorema III. - Se un punto $Z$ di coordinate $\zeta_{0}, \zeta_{1}, \ldots, \zeta_{n} \dot{e} \eta_{i}^{p l o}$ per l'ipersuperficie $F_{i}=o$ e $\theta_{i}^{\text {plo }}$ per la $\varphi_{i}=0$, e se è nullo almeno uno dei numeri $n_{0}, n_{1}, \ldots, n_{b}$, $\theta_{0}, \theta_{1}, \ldots, \theta_{k}$, essendo peró diverse da zero o tutte le $n_{i}$ o tutte le $\theta_{i}$, ossia se, per fissare le idee, le $n_{0}, n_{1}, \ldots, n_{b}$ sono tutte diverse da zero $e \theta_{0}, \theta_{1}, \ldots, \theta_{k^{\prime}}$ sono le $\theta_{i}$ non nulle, essendo $k^{\prime} \leq k-\mathrm{I}$, allora la varietà $W$ ba in $Z$ un punto di molteplicità

$$
\left\{\begin{array}{r}
V_{n-b-k+1}\left(n_{0}-\mathrm{I}, \ldots, n_{b}-\mathrm{I}, \theta_{0}-\mathrm{I}, \ldots, \theta_{k^{\prime}}-\mathrm{I}\right) \\
+V_{n-b-k}\left(n_{0}-\mathrm{I}, \ldots, n_{b}-\mathrm{I}, \theta_{0}-\mathrm{I}, \ldots, \theta_{k^{\prime}}-\mathrm{I}\right) .
\end{array}\right.
$$

La varietà $\Theta$, luogo delle tangenti in $Z$ alla $W$, è di ordine dato dalla (5) ed è rappresentata nelle coordinate correnti omogenee di punto $y_{0}, y_{1}, \ldots, y_{n}$ coll'annullare la matrice

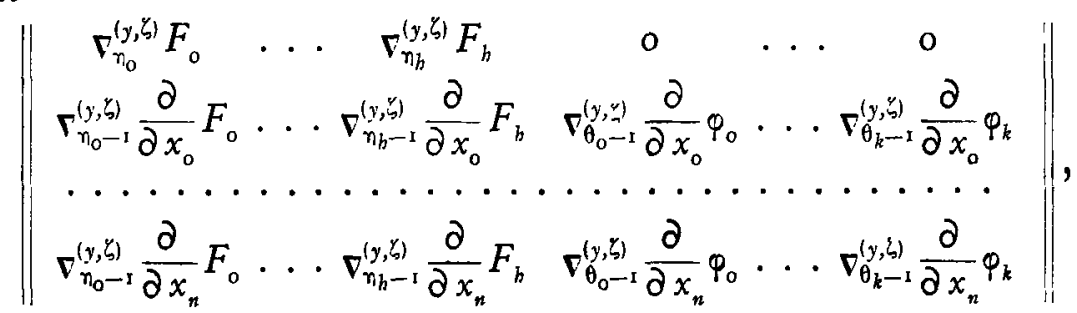

dove l'operazione $\Gamma_{i}^{(y, \zeta)}$ per $i \supseteq$ I significa che si deve prima eseguire l'operazione $\nabla_{i}^{(y, x)}$ e poi sostituire le $\zeta_{0}, \zeta_{1}, \ldots, \zeta_{n}$ coordinate di $Z$ alle $x_{0}, x_{1}, \ldots, x_{n}$, per $i=0$, $i=-$ I significa che si debbono sostituire $l e \zeta_{0}, \zeta_{1}, \ldots, \zeta_{n}$ alle $x_{0}, x_{1}, \ldots, x_{n}$.

Se tutte le $n_{i}, \theta_{i}$ sono diverse da zero, allora la dimensione della varietà rappresentata coll'annullare la matrice (6) è $b+k$ e non $b+k-\mathrm{I}$, onde sard conveniente 
pensare la $W$ rappresentata analiticamente annullando una qualunque delle matrici $(3)_{i}$ $(i=0, \mathrm{I}, \ldots, n)$, dove s'indica con $(3)_{i}$ la matrice ottenuta dalla (3), quando nella linea $(i+2)^{\text {sima }}$ si ponga

in luogo di

$$
\frac{p F_{0}}{x_{i}} \quad \frac{p F_{1}}{x_{i}} \ldots \frac{p F_{b}}{x_{i}} \quad \frac{q \varphi_{0}}{x_{i}} \quad \frac{q \varphi_{1}}{x_{i}} \ldots \frac{q \varphi_{k}}{x_{i}}
$$

$$
\frac{\partial}{\partial x_{i}} F_{0} \quad \frac{\partial}{\partial x_{i}} F_{1} \ldots \frac{\partial}{\partial x_{i}} F_{b} \quad \frac{\partial}{\partial x_{i}} \varphi_{0} \quad \frac{\partial}{\partial x_{i}} \varphi_{1} \ldots \frac{\partial}{\partial x_{i}} \varphi_{k} .
$$

Scelta tra queste matrici per es. la (3), per fissare le idee, segue che per ottenere la rappresentazione analitica della varietà $\Theta$, luogo delle tangenti in $Z$ alla $W$, si dovranno eseguire le operazioni seguenti :

$\Gamma_{a}^{(y, \zeta)}$ sui determinanti di ordine $b+k+2$, che non contengono nè la $I^{2}$ nè la $2^{\mathrm{a}}$ linea della $(3)_{0}$,

$\nabla_{a+1}^{(y, b)}$ su quelli che contengono o la $\mathrm{I}^{\mathrm{a}}$ o la $2^{\mathrm{a}}$ linea, ma non entrambe della (3), essendo

$\boldsymbol{r}_{a+2}^{(y, b)}$ su quelli, che contengono entrambe le prime due linee della $(3)_{0}$,

$$
a=n_{0}+n_{1}+\cdots+n_{h}+\theta_{0}+\theta_{1}+\cdots+\theta_{k}-(b+k+2) .
$$

Si trae quindi:

Teorema IV. - Se tutte le $n_{i}$ e le $\theta_{i}$ sono diverse da zero, e inoltre o non si ba $n_{0}=n_{1}=\cdots=n_{h}$, oppure non si ba $\theta_{0}=\theta_{1}=\cdots=\theta_{k}$, allora la varietà $W$ ba in $Z$ un punto di molteplicità

$$
\left\{\begin{array}{r}
V_{n-b-k+1}\left(n_{0}-\mathrm{I}, \ldots, n_{b}-\mathrm{I}, \theta_{0}-\mathrm{I}, \ldots, \theta_{k}-\mathrm{I}\right) \\
+2 V_{n-b-k}\left(n_{0}-\mathrm{I}, \ldots, n_{b}-\mathrm{I}, \theta_{0}-\mathrm{I}, \ldots, \theta_{k}-\mathrm{I}\right) \\
+V_{n-b-k-1}\left(n_{0}-\mathrm{I}, \ldots, n_{b}-\mathrm{I}, \theta_{0}-\mathrm{I}, \ldots, \theta_{k}-\mathrm{I}\right) .
\end{array}\right.
$$

La varietà $\Theta$, luogo delle tangenti in $Z$ alla $W$, è di ordine dato dalla (7) ed è rappresentata nelle coordinate correnti $y_{0}, y_{1}, \ldots, y_{n}$ coll'annullare una qualunque delle matrici $(6)_{i}\left(i=0,1, \ldots, n\right.$, purchè sia $\left.\zeta_{i} \neq 0\right)$, indicando con $(6)_{i}$ la matrice che si ottiene dalla $(6)$, ponendo nella linea $(i+2)^{\text {sima }}$

in $\operatorname{luog} 0 \mathrm{di}$

$$
\frac{p}{\zeta_{i}} \nabla_{\eta_{0}}^{(y, \zeta)} F_{o} \ldots \frac{p}{\zeta_{i}} \nabla_{n_{b}}^{(y, \zeta)} F_{h} \quad \sum_{\zeta_{i}}^{q} \nabla_{\theta_{0}}^{(y, \zeta)} \varphi_{0} \ldots \frac{q}{\zeta_{i}} \nabla_{\theta_{k}}^{(y, \zeta)} \varphi_{k}
$$

$$
\Gamma_{n_{0}-1}^{(y, \zeta)} \frac{\partial}{\partial x_{i}} F_{0} \ldots \Gamma_{n_{b}-1}^{(y, \zeta)} \frac{\partial}{\partial x_{i}} F_{b} \quad \Gamma_{\theta_{0}-1}^{(y, \zeta)} \frac{\partial}{\partial x_{i}} \Psi_{0} \ldots \Gamma_{\theta_{k}-1}^{(y, \zeta)} \frac{\partial}{\partial x_{i}} q_{k} .
$$

Quando è $n_{0}=n_{1}=\cdots=n_{b}>0, \theta_{0}=\theta_{1}=\cdots=\theta_{k}>0$, allora la dimensione della varietà rappresentata coll'annullare una qualunque delle matrici $(6)_{i}$ è $b+k$ e non $b+k-1$. Infatti, posto $n=n_{0}=n_{1}=\cdots=n_{1}, \theta=\theta_{0}=\theta_{1}=\cdots=\theta_{k}$, affinchè sia nulla la matrice $(6)_{i}$ basta che sia nulla la matrice $\left(6^{\prime}\right)_{i}$, che si ottiene dalla (6) moltiplicando per $y_{i}$ gli elementi della linea $(i+2)^{\text {sima }}$ della stessa matrice 
(6), perchè si ha:

$$
\begin{aligned}
\frac{n}{\zeta_{i}} \nabla_{n_{j}}^{(y, \zeta)} F_{j}=\frac{1}{\zeta_{i}} \sum_{u=0}^{u=n} y_{u} \nabla_{\eta_{j-1}}^{(y, \zeta)} \frac{\partial}{\partial x_{u}} F_{j} & (j=0, \mathrm{I}, \ldots, h), \\
\frac{\theta}{\zeta_{i}} \nabla_{\theta_{j}}^{(y, \zeta)} \varphi_{j}=\frac{1}{\zeta_{i}} \sum_{u=0}^{u=n} y_{u} \nabla_{\theta_{j-1}}^{(y, \zeta)} \frac{\partial}{\partial x_{u}} \varphi_{j} & (j=0, \mathrm{I}, \ldots, k) .
\end{aligned}
$$

Seguendo un metodo analogo a quello usato per dedurre il teorema IV, si puó costruire l'equazione della varietd $\theta$, quando è $n_{0}=n_{1}=\cdots=r_{h}, \theta_{0}=\theta_{1}=\cdots=\theta_{k}$, e si trova:

TeOrema V. - Se $n_{0}=n_{1}=\cdots=n_{h}=n>0, \theta_{0}=\theta_{1}=\cdots=\theta_{k}=\theta>0$, allora la varietà $W$ ba in $Z$ un punto di molteplicità

(8)

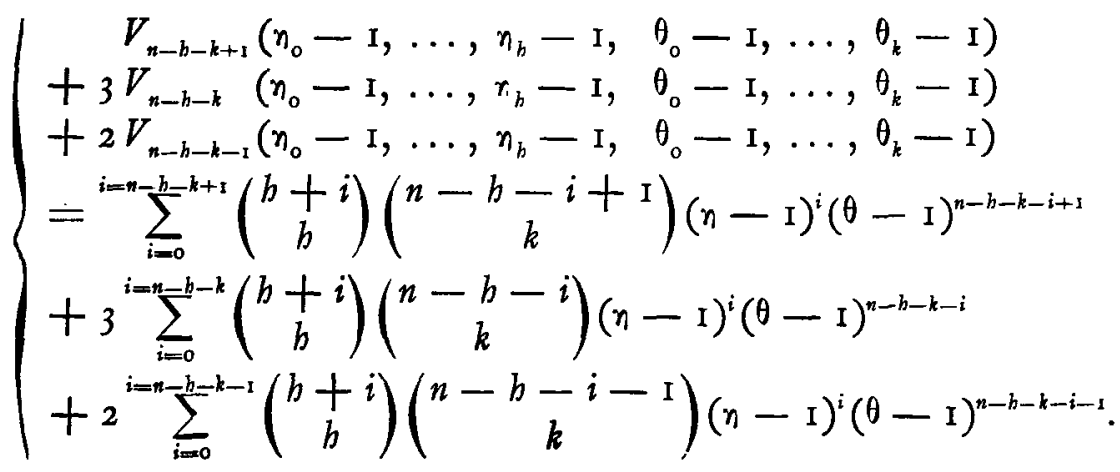

La varietà $\Theta$, luogo delle tangenti in $Z$ alla $W, \dot{e}$ di ordine dato dalla (8).

Quando in particolare uno dei dati sistemi lineari (I), (2) è un sistema lineare d'iperpiani, i nostri risultati dànno luogo ai teoremi VIII, IX, X, XII del citato lavoro del Lorenzola.

Torino, marzo 1907.

Giovanni Z. Giambelli. 